\title{
The multiple spirals in the disk of HD100546
}

\author{
A. Boccaletti ${ }^{1}$, A.-M. Lagrange ${ }^{2}$, E. Pantin ${ }^{3}$, J.-C. Augereau ${ }^{2}$ \\ S. P. Quanz ${ }^{4}$ and H. Meheut ${ }^{3}$ \\ ${ }^{1}$ LESIA, Observatoire de Paris, 5 place Jules Janssen, 92195 Meudon, France \\ email: anthony.boccaletti@obspm.fr \\ ${ }^{2}$ IPAG, Université Joseph Fourier, CNRS, BP 53, 38041 Grenoble, France \\ ${ }^{3} \mathrm{SAp}, \mathrm{CEA}$, France \\ ${ }^{4}$ Institute for Astronomy, ETH Zurich, Wolfgang-Pauli-Strasse 27, 8093 Zurich, Switzerland
}

\begin{abstract}
We report the detection of multi-armed spirals in the environment of HD 100546 using NICI at Gemini South in the Ks band. These data feature a better angular resolution and higher contrast than previous HST images, which allows to resolve the former known spiral into a multiple pattern. An analytic model with a gravitational perturber is used to fit the spiral pattern. We derived limit of detections which set constraints on the discovered forming planet.
\end{abstract}

Keywords. stars: individual (HD 100546) - protoplanetary disks - high angular resolution

\section{Introduction}

The Herbig Be star HD 100546 (5-10 Myr, 97 pc) is known to harbour a pre-transitional protoplanetary disks already imaged with other instruments. Using NICMOS on HST the disk was seen as an elliptical pattern without obvious structure in the near IR (Augereau et al. (2001)), but the image from Ardila et al. (2007), owing to an improved angular resolution at visible wavelengths, revealed a much more complex morphology. The disk region between $1.5-3$ " is of particular interest since it contains two trailing spiral arms developing towards the south-east and the north-west respectively. The innermost part of the disk intensively studied by Benisty et al. (2010) with the VLTI suggests an inner hole between 4 and $13 \mathrm{AU}$ indicative of an inner giant planet. Recently, Quanz et al. (2013) reported the discovery of a planet forming candidate.

\section{The spirals of HD 100546}

We retrieved archival data obtained with the Near Infrared Coronagraphic Imager (NICI), installed at Gemini South. The observation were done on March 6th, 2010, using a single channel configuration in the Ks band $(2.15 \mu \mathrm{m})$ with the 0.32 " coronagraphic mask and with the telescope pupil at a constant orientation to allow for angular differential imaging (ADI). We followed the data reduction procedure described in Boccaletti et al. (2013) using various ADI flavours like KLIP and LOCI. While the large scale component of the disk is removed by ADI, a complex set of spiral patterns is detected in the southeast part of the field in the place where Ardila et al. (2007) reported the detection of a single spiral. The spirals are labeled in Fig. 1 from S1 to S6. S1 is the brightest and longest spiral, it starts from $\rho=1.40 ", P A=374^{\circ}$ to $\rho=2.27^{\prime \prime}, P A=329^{\circ}$. The equivalent projected length is about $155 \mathrm{AU}$. Both S1 and S2 are seen in the ACS/HST image as a single broken spiral. The image was un-projected assuming an inclination of $42^{\circ}$ and the spines of the spirals were fitted with the Muto et al. (2012) model, which assumes a gravitational perturber launching a single spiral pattern in a gaseous disk. On average for all spirals, 

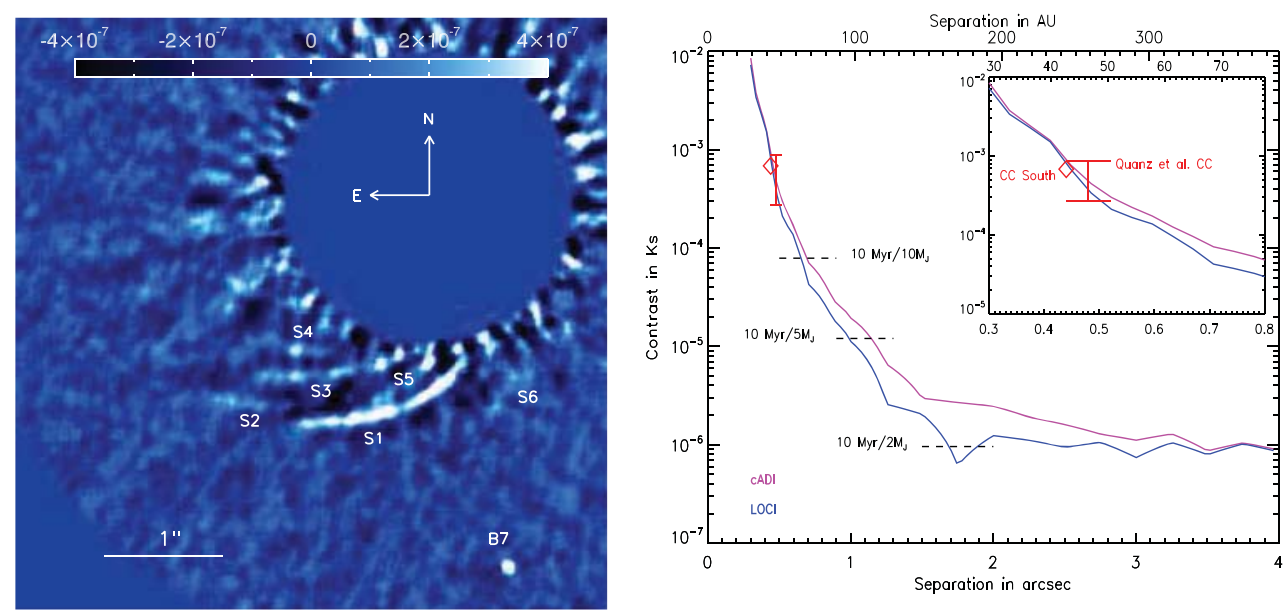

Figure 1. Left: Image of the HD 100546 environment processed with LOCI. The spirals are labeled S1 to S6. Right: Limits of point source detection estimated with ADI and LOCI using fake planets to calibrate the ADI self-subtraction. The expected intensity of the forming planet (Quanz et al. CC) is reported as well as a new candidate identified in NICI image (CC south).

we obtained $r_{c}=0.23 \pm 0.04 ", \theta_{0}=230 \pm 55^{\circ}$, and $h c=0.13 \pm 0.04$, with $r_{c}$ and $\theta_{0}$ the polar coordinates of the perturber and $h c$ the disk aspect ratio. However, we note that the parameters are highly degenerated. If one or several perturbers are responsible for these spirals, the model of Muto et al. (2012) derives a physical separation of $20-30 \mathrm{AU}$ from the star, which is out of the gap detected from interferometry and closer than the location of the forming planet. Using a fake spiral injected at the northwest, we were able to measure the width and photometry of S1 as well as a lower limit of the anisotropic scattering factor $(g>0.48-0.61)$ which is derived from the non detection of the northwest spiral. In addition, we did not detect in Ks any counter part of the Quanz et al. (2013) forming planet. As a result, the contrast (Fig. 1, right) achieved in the NICI data at a separation of 0.48 " allows to rule out a self-luminous planet more massive than $16-18 \mathrm{M}_{J}$ according to the age and whatever the evolutionary model. Quanz et al. (2013) suggested that if the planet is still forming the flux measured in Lp may not entirely come from its photosphere

Any large perturbation in a disk of gas tends to launch sound waves resulting in a spiral shape due to the differential rotation of the disk. Several scenarios can be considered to account for the presence of spirals. A protoplanetary disk can also undergo gravitational instabilities. But in the case of HD 100546, the estimation of the Toomre parameter, based on currently estimated disk's mass as well as the disk aspect ratio, is well above unity. We concluded that gravitational instabilities are unlikely to account for the presence of spirals and the effect of gravitational perturbers is preferred. More detailed analysis will be presented in a forthcoming paper.

\section{References}

Augereau, J. C., Lagrange, A. M., Mouillet, D., \& Menard, F., 2001, A\&A, 365, 78

Ardila, D. R., Golimowski, D. A., Krist, J. E., et al., 2007, ApJ, 365, 78

Benisty, M., Tatulli, E., Menard, F., \& Swain, M. R., 2010, A\&A, 511, 75

Boccaletti, A., Lagrange, A. M., Bonnefoy, M., Galicher, R., \& Chauvin, G., 2013 A $\& A, 551,14$

Muto, T., Grady, C. A., Hashimoto, J., Fukagawa, M. et al., 2012, ApJ, 748, 22

Quanz, S. P., Amara, A., Meyer, M. R., et al., 2013 ApJL, 766, 1 